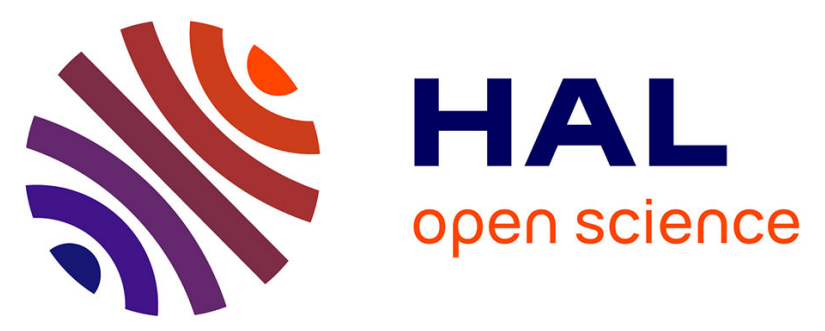

\title{
Electrophysiological brain mapping: Basics of recording evoked potentials induced by electrical stimulation and its physiological spreading in the human brain
}

Marion Vincent, David Guiraud, Hugues Duffau, Emmanuel Mandonnet, François Bonnetblanc

\section{To cite this version:}

Marion Vincent, David Guiraud, Hugues Duffau, Emmanuel Mandonnet, François Bonnetblanc. Electrophysiological brain mapping: Basics of recording evoked potentials induced by electrical stimulation and its physiological spreading in the human brain. Clinical Neurophysiology, 2017, 128 (10), pp.18861890. 10.1016/j.clinph.2017.07.402 . hal-01613032v2

\section{HAL Id: hal-01613032 \\ https://hal-lirmm.ccsd.cnrs.fr/hal-01613032v2}

Submitted on 24 Oct 2017

HAL is a multi-disciplinary open access archive for the deposit and dissemination of scientific research documents, whether they are published or not. The documents may come from teaching and research institutions in France or abroad, or from public or private research centers.
L'archive ouverte pluridisciplinaire HAL, est destinée au dépôt et à la diffusion de documents scientifiques de niveau recherche, publiés ou non, émanant des établissements d'enseignement et de recherche français ou étrangers, des laboratoires publics ou privés. 


\section{Electrophysiological brain mapping: basics of recording evoked potentials induced by electrical stimulation and its physiological spreading in the human brain.}

Marion Vincent¹, David Guiraud1, Hugues Duffau4, Emmanuel Mandonnet5,, ${ }^{4}$ François Bonnetblanc ${ }^{1,2,3,{ }^{*},}$

${ }^{1}$ INRIA, Université de Montpellier, LIRMM, équipe Camin, F-34095 Montpellier, France.

${ }^{2}$ Cognition, Action et Plasticité Sensorimotrice, INSERM U1093, Université de Bourgogne, UFR STAPS, F-27877 Dijon, France.

${ }^{3}$ Institut Universitaire de France, F-75005 Paris, France.

${ }^{4}$ Département de Neurochirurgie, Hôpital Gui de Chauliac, F-34295 Montpellier, France; Institut des Neurosciences de Montpellier, INSERM U1051, Hôpital Saint Eloi, F-34091 Montpellier, France.

${ }^{5}$ Assistance Publique-Hôpitaux de Paris (AP-HP), Service de Neurochirurgie, Hôpital Lariboisière, Paris, France. IMNC, UMR8165, Orsay, France. Université Paris 7 Diderot, Paris, France.

${ }^{*}$ Both authors contributed equally to this work, corresponding authors: emmanuel.mandonnet@aphp.fr and francois.bonnetblanc@inria.fr

Conflict of interest: None. 


\section{Introduction}

Being able to change or inhibit the activity of a region or population of neurons in the brain is an essential approach in fundamental neuroscience, as it helps the researcher to determine the functional role of neurons. This approach is also important at a more applied level, for brain function mapping during neurosurgical procedures (Desmurget et al. 2007). It is well known that electrical stimulation (ES) affects neural activity by modifying the voltage gradient along the neuronal cell inducing depolarization or hyperpolarization of the membrane. When a current flows in tissues around neuronal cells, it can change their membrane potential and trigger an action potential. However, this general principle can be applied in vivo via several different settings and much is unknown about which neural elements are excited or inhibited locally (Ranck 1975) and how this local perturbation spreads within the brain through physiological pathways (Vincent et al. 2016a). We briefly present here different types of electrophysiological potentials that are evoked by ES in the human brain and some basic methodological considerations required for their correct assessment. Three different types of evoked potentials should be distinguished:

- Cortical evoked-potential (also called direct cortical response, DCR), when recording the cortex at the stimulation site,

- Cortico-axono-cortical evoked-potential, i.e. recording the cortex at a distant site from the stimulating site. These potentials are elicited by physiological propagation through white matter associative pathways from the locally stimulated area towards the distal area,

- Axono-cortical evoked potentials, when the cortex is distally recorded from a stimulation site within the white matter. 
These evoked potentials are technically difficult to observe. Their recording imposes important methodological considerations about the way they can be triggered and measured. In particular, we detail the factors potentially determining the generation of true cortico-axono-cortical evoked potentials, spreading from one stimulated cortical area to another distant one and passing through the white matter pathways. We further offer an explanation about why a current frequency of $50-60 \mathrm{~Hz}$ is needed to obtain an effect at the behavioural level. Finally, we propose a simple theory suggesting how ES hyperpolarizes a cortical area, thus excluding this area from the network it belongs to. As a result, the function is not anymore supported by this disconnected network. ES only perturbs locally a single area within a network of several cortical areas "dialoguing" together and thus perturbs its functional connectivity.

\section{Methodological pitfalls when measuring evoked potentials in the brain}

Measuring evoked potentials triggered by ES in the brain is somehow a difficult challenge. Some requirements are needed in order to verify and check that the putative variations in the voltage difference between the recording electrodes and their reference is not due to stimulus and filtering artefacts, which are the consequences of the electronic responses of the measuring system. Two requirements are quite simple, but surprisingly never discussed before in the literature. First, the validity of any evoked potential can be verified if the current polarity, even if it is a biphasic current, is reversed, for instance by switching the poles of the probe, thus reversing the anode and the cathode. This simple manipulation allows verifying that the polarity of true physiological potentials is not reversed, whereas that of ES artefact is. In most cases, biphasic current has been alternated mainly to cancel the stimulation artefacts when averaging several trials together. However, by averaging, one cannot determine the 
electronic noise induced by the system, thus precluding to measure the evoked potential on a trial-by-trial basis.

In particular, caution should be taken with respect to oscillations that can appear due to the filter step response of the amplifier. In this case, it is important to analyse the data on a trial-by-trial basis in order to accurately identify and remove this unphysiological oscillation. Averaging several trials together without a detailed analysis of the raw signals can clearly mask those oscillations and lead to false interpretations (Vincent et al. 2016b).

\section{Stimuli and recording parameters for the DCR and the ACEP}

Usual cortical and white matter functional mappings were first completed on awake patients using a current controlled stimulator (Nimbus, Captomed, France). DES was delivered thanks to a bipolar probe ( $0.5 \mathrm{~mm}$ diameter electrode tips, $5 \mathrm{~mm}$ apart). Constant-current biphasic square wave pulse of $1 \mathrm{~ms}$ duration each was applied at a fixed frequency of $60 \mathrm{~Hz}$. The current intensity was patient-specific within 1.5 to $2 \mathrm{~mA}$. EPs recordings were performed after the tumour resection, under general anaesthesia, according to the following detailed set-up.

4-contacts strips of ECoG electrodes ( $4 \mathrm{~mm}$ exposed surface stainless steel contacts, 10 mm spaced, DIXI, France) were positioned on the surface of the brain. ECoG signals were recorded using differential (contacts 1 to 3 and 5 to 7 ) and common mode (contacts 4 and 8) configurations. For the differential mode, both active and reference are two adjacent electrodes of one strip. The signal was measured between each channel of interest (active) and a reference electrode for the classical common mode configuration. The reference electrode (Au cupule-electrode, G.tec, Austria) was located on the mastoid 
ipsilateral to the surgery. All channels were grounded to a patch-electrode located on the same-side acromion.

ECoG signals were amplified and filtered (g.BSamp, G.tec, Austria) before being digitally converted (PowerLab, ADInstrument). A band-pass filter was set to [0.5 Hz - $1 \mathrm{kHz}]$ as it allows acquiring all useful information of ECoG. To minimize the saturation of the frontend amplifier, signals were recorded with a gain of 1,000. Using the $50 \mathrm{~Hz}$ Notch filter integrated in the amplifier was required for all the recordings to ease the impact of the electrical noise present in the operative room. Unused amplifier's channels were bypassed. Finally, data were sampled at $10 \mathrm{kHz}$.

Low-frequency DES was performed at different cortical and sub-cortical sites, identified as functional during the brain mapping. Stimulation intensities were adapted to each patient and to the type of evoked potentials to record. DCRs were recorded with 5 and $10 \mathrm{~Hz}$ DES, applied with intensities ranging from 0.5 to $2 \mathrm{~mA}$. ACEPs were induced by 1 mA DES at $5 \mathrm{~Hz}$.

\section{The various types of potentials evoked by electrical stimulation.}

Three different categories of cortical evoked potentials can be measured, depending on the location of the recording electrodes relatively to the stimulating electrode.

The DCR is an electrical potential recorded in the immediate vicinity (distance to the stimulation $<2-5 \mathrm{~mm}$ ) of a focal electrical stimulation applied on the cortical surface (Adrian 1936, Goldring et al. 1991, 1994, Vincent et al. 2016b). This potential can either be negative or positive depending on the recording mode. For the classical common mode configuration, the signal is measured between each active electrode and a distant reference electrode or an average of all electrodes' signals and the DCR appears to be 
negative. For a differential recording mode in which an adjacent electrode serves as reference for the nearest electrode in order to improve the focality, the DCR can either be negative or positive depending on the location of the potential towards the active and reference electrodes (Vincent et al. 2016b). Its amplitude peaks between 15-25 ms after the beginning of the stimulation. A typical DCR is presented in Figure 1.

Please insert Figure 1 here

It has been suggested that the DCR depends mainly on post-synaptic events. A weak stimulus evokes a $20 \mathrm{~ms}$ negative deflection called the primary negative potential that represents excitatory post-synaptic potentials of apical dendrites (Goldring et al. 1994). In addition, it is also assumed that the DCR has different profiles that are correlated to the local cytoarchitectonic organisation of the stimulated area (Goldring et al. 1994). They also could be used to establish in real-time and in vivo the electrophysiological state of a particular area as for instance the level of excitability (Vincent et al. 2016b)

The ACEP is also an electrical potential recorded on the cortical surface (Mandonnet et al. 2016, Yamao 2014), but obtained when the subcortical white matter pathways are directly stimulated in the cavity (e.g. after resection of a brain tumor). As for DCR, this potential can either be positive or negative depending on the recording mode. In theory it could be recorded quite far from the site of ES, however a single proof of an ACEP has been obtained $\sim 3 \mathrm{~cm}$ further from the site of stimulation (Yamao et al. 2014). For similar parameters, its amplitude peaks before that of a DCR and around 10-25 ms after the beginning of the stimulation. A typical ACEP is presented in Figure 2. 
Please insert Figure 2 here

The true cortico-cortical evoked potential (CCEP), that should rather be named corticoaxono-cortical evoked potential (CACEP), is a post-synaptic potential recorded on the cortical surface. It is obtained when a distant cortical area is stimulated (Matsumoto et al. 2004 and Keller et al. 2011). This type of potential might be more difficult to measure in the context of intraoperative brain mapping performed with the standard bipolar probe. It has mainly been described in the context of epilepsy when two adjacent electrodes of subdural electrocorticographic grids (ECoG) were used to produce a bipolar stimulation (space between the two poles: $\sim 10 \mathrm{~mm}$ ) and for high intensity (around $10 \mathrm{~mA}$ ). We do not present a typical CACEP since there is currently no raw and single measurement of one sample without averaging of a large amount of trials together (see previous methodological considerations). As reported above, in all this literature, the main requirements for evoked potential verification were not identified and discussed adequately.

\section{How to obtain true CACEP?}

As mentioned previously, CACEPs have only been measured in the context of epilepsy (Matsumoto et al. 2004, Keller et al. 2011), when ES was applied thanks to subdural grids and two contacts were used as a cathode and an anode for bipolar stimulations. Such CACEPs have been not yet reported when stimulating with a classical $5 \mathrm{~mm}$ spaced bipolar probe. The comparison between these two modes of ES is important and yields a main hypothesis to explain why it could be more difficult to observe CACEPs with a 
classical $5 \mathrm{~mm}$ spaced bipolar probe rather than with grids electrodes. In the former situation, ES may not reach the deeper layers of the cortical columns due to insufficient intensity. To overcome this issue, the biophysics of ES suggest (i) increasing the interelectrode distance and (ii) increasing stimulus intensity.

Indeed, for bipolar ES applied on the cortical surface, the depth of the electrical field is determined by the inter-electrode distance. The longer the inter-electrode distance is, the deeper in the cortex the electrical field is. In this vein, it has been demonstrated that large-diameter axons in the subcortex are the most excitable neural elements, when electrical fields are generated and extend from the cortical surface to the subcortical white matter (Landau et al. 1965). In addition, when the delivered current intensity is increased, as the medium is almost linear, the deeper electrical field is also increased. This interpretation is illustrated in Figure 3.

Two other important aspects should also be taken into account to better understand the generation of CACEPs. First, with a monopolar probe, electrical charges spread over longer distance from the source, so it may be possible that this mode of ES could generate CACEPs with a lower charge injection but with a lower focalisation. We limited our considerations here to situations where a bipolar probe is used for a more accurate functional brain mapping. Second, it has been demonstrated experimentally and theoretically through modelling, that the generation of action potentials in an axon is obtained with less charge injections when the pulse width (PW) is shorter than the chronaxy (McCreery et al. 1990, Merrill et al. 2005). The relationship between the charges injected to reach the threshold and the PW is not flat but roughly an affine function with a positive slope (see figure 7 in page 7 of Vincent et al. 2016a). The more 
the PW decreases the more the required charges to inject to reach the threshold decrease.

So hypothetically, reducing the PW could also facilitate the generation of CACEPs. Interestingly, in the literature presenting CACEPs measured with grids of $1 \mathrm{~cm}$-spaced electrode, PW are inferior to that used commonly during intraoperative mapping with 5 mm-spaced bipolar Ojemann's stimulation. Further studies should disambiguate the effect of the PW and the inter-electrodes spacing.

\section{Why a 50-60 Hz ES current is used for brain mapping?}

It is currently not clear why $50-60 \mathrm{~Hz}$ ES is needed to perform functional mapping of the brain during neurosurgery. Interestingly, it has been demonstrated that with $20 \mathrm{~Hz}$ ES slow negativities of the separated DCR of a series fuse to produce a negative shift of the cortical electrophysiological baseline level. As such a shift develops, the sequentially DCRs are reduced in amplitude (see Goldring et al. 1961). It is thus tempting to suggest that when the frequency of stimulation is increased, a progressive hyperpolarisation is locally induced around the site of stimulation that may perturb the functioning of this area. Indeed, this slow negative shift of the baseline with attenuation of the DCRs may be representative of a global hyperpolarisation (and not a depolarisation) because no output response is generated (unless the intensity is much increased to trigger more directly an all or none somatic response, see figure 2 of Goldring et al. 1994 and its interpretation). More precisely, it may illustrate the fact that the membranes of certain neural elements which are under the ES do not have an all or none property but saturate and did not recover their resting state due to the cumulating DCRs. In this vein and importantly, the duration of the DCR is 15 to $20 \mathrm{~ms}$, which corresponds to the period of the 50-60 Hz ES. Thus, when successive ES are applied too frequently, the cortex may 
not respond due to the saturation of the membranes occurring during the cumulating cortical responses.

\section{A simple hypothesis of the local cortical perturbation effect of ES within the functional networks.}

When classical parameters of ES are used for intraoperative functional mapping (1-2 mA current, bipolar probe with $5 \mathrm{~mm}$ between the two poles, biphasic current, $1 \mathrm{~ms}$ pulse width, 50-60 Hz frequency) there may be no physiological spreading of evoked action potentials to distant sites. By contrast, $60 \mathrm{~Hz}$ ES would perturb only and locally the functioning of the most superficial cortical columns. By hyperpolarizing them below the basal electrophysiological level (see previous part and Goldring et al. 1961), they would be kept silent and functionally disconnected from other cortical areas. They would be unresponsive to the afferent inputs and would not send action potentials to other areas. As a consequence, their deactivation could disrupt the functional connectivity of the whole network they belong to, and could induce the behavioural perturbations that are observed during awake brain surgeries. A sort of phase desynchronization between the different cortical nodes could be involved, preventing integration and segregation between various areas and the efficacy of the functional connectivity within a given network (Vincent et al. 2016a, Varela et al. 2001).

Please insert Figure 3A and 3B here

This 50-60 Hz ES focal hyperpolarization - functional disconnection hypothesis could explain 'negative' clinical effects like aphasia, dysphasia, or motor inhibition when 
stimulating association cortices. However, it appears to explain less well the 'positive' clinical effects like focal tonic-clonic movement, paresthesiae or phosphines commonly observed when stimulating primary motor, sensory or visual cortices. In these latter cases, there may be an indirect disinhibition of pyramidal neurons, through the ESinduced inhibition. This disinhibition could depend also on many architectonic factors of the stimulated areas, including the role of local inhibitory networks and the way a given area integrates successive ES pulses (electrophysiological response to the frequency modulation). Further efforts should be made to better understand this fascinating phenomenon.

Correctly measuring evoked potentials in the human brain induced by electrical stimulation is important in the clinical domain especially in the neurosurgical context. It remains challenging because of many pitfalls that can occur at the methodological level and few teams in the world are currently able to efficiently record these evoked potentials. Nevertheless, they can give strong real-time in-vivo insights into the functional state and connectivity of a patient's brain.

In the next years measuring intraoperatively the evoked potentials with ES in the brain will be a new method for mapping the brain in vivo and in real time and taking into account the specificity of each patient's brain.

Acknowledgments: We thank Pr David B. MacDonald for his precise and stimulating comments about the present work. 


\section{References}

Adrian ED (1936) The spread of activity in cerebral cortex. J Physiol 88: 127-161.

Desmurget M, Bonnetblanc F, and Duffau H (2007) Contrasting acute and slow-growing lesions: a new door to brain plasticity. Brain 130: 898-914.

Goldring S, Jerva MJ, Holmes TG, O’Leary JL, Shields JR (1961) Direct response of human cerebral cortex. Arch Neurol 4:22-30.

Goldring S, Harding GW, Gregorie EM (1994) Distinctive electrophysiological characteristics of functionally discrete brain areas: a tenable approach to functional localization. J Neurosurg 80:701-709.

Histed, M.H., Bonin, V., and Reid, R.C. (2009). Direct activation of sparse, distributed populations of cortical neurons by electri- cal microstimulation. Neuron 63, 508-522.

Keller, C.J., Bickel, S., Entz, L., Ulbert, I., Milham, M.P., Kelly, C., and Mehta, A.D. (2011). Intrinsic functional architecture predicts electrically evoked responses in the human brain. Proc. Natl. Acad. Sci. USA 108, 10308-10313.

Landau WM, Bishop GH, Clare MH (1965) Site of excitation in stimulation of the motor cortex. J Neurophysiol 28:1206-1222.

Mandonnet E, Dadoun Y, Poisson I, Madadaki C, Froelich S, Lozeron P (2016) Axonocortical evoked potentials: a proof-of-concept study. Neurochirurgie 62:67-71.

Matsumoto, R., Nair, D.R., LaPresto, E., Najm, I., Bingaman, W., Shibasaki, H., and Lüders, H.O. (2004) Functional connectivity in the human language system: a corticocortical evoked potential study. Brain 127, 2316-2330. 
McCreery DB, Agnew WF, Yuen TGH, Bullara LA (1990) Charge density and charge per phase as cofactors in neural injury induced by electrical stimulation. IEEE Trans Biomed Eng ;37:996-1001

Merrill, D.R., Bikson, M., and Jefferys, J.G.R. (2005). Electrical stimulation of excitable tissue: design of efficacious and safe protocols. J. Neurosci. Methods 141, 171-198.

Ranck, J. B. (1975). Which elements are excited in electrical stimulation of mammalian central nervous system: a review. Brain research, 98(3), 417-440.

Varela F, Lachaux JP, Rodriguez E, Martinerie J (2001) The brainweb: phase synchronization and large-scale integration. Nat Rev Neurosci 2(4):229-39.

Vincent M, Rossel O, Hayashibe M, Herbet G, Duffau H, Guiraud D, Bonnetblanc F (2016a) The difference between electrical microstimulation and direct electrical stimulation towards new opportunities for innovative functional brain mapping? Rev Neurosci 27:231-58.

Vincent M, Rossel O, Duffau H, Bonnetblanc F, Guiraud D (2016b) A measure of corticocortical potentials evoked by $10 \mathrm{~Hz}$ direct electrical stimulation of the brain and by means of a differential recording mode of electrocorticographic signals. Conf Proc IEEE Eng Med Biol Soc 4543-4546.

Yamao Y, Matsumoto R, Kunieda T, Arakawa Y, Kobayashi K, Usami K, Shibata S, Kikuchi T, Sawamoto N, Mikuni N, et al. (2014) Intraoperative dorsal language network mapping by using single-pulse electrical stimulation. Human brain mapping, 35(9):4345-4361. 
Figure captions

Figure 1. ECoG signal corresponding to the stimulation of a cortical site close to the electrode (gain $=10^{4}$ ). A raw negative DCR (no averaging) can be observed $\sim 25 \mathrm{~ms}$ systematically after the three stimulation artefacts. Current Intensity $=0.5 \mathrm{~mA}$ and ES frequency $=5 \mathrm{~Hz}$. Identical DCR were also observed when the polarity of the ES was reversed. Note that a constant and recurrent noise is present here probably due to the scialytic operation lights. Electrophysiological measurement in the operating room is a challenging task. Measures were made using a differential recording mode (Vincent et al. 2016b).

Figure 2. ECoG signal corresponding to the stimulation of subcortical white matter pathways about $2 \mathrm{~cm}$ from the electrode (gain $=10^{4}$ ). A raw positive ACEP (no averaging) can be observed $\sim 25 \mathrm{~ms}$ after the stimulation artefact. Current Intensity $=$ $1 \mathrm{~mA}$ and ES frequency $=5 \mathrm{~Hz}$. Identical ACEP were also observed when the polarity of the ES was reversed. Measures were made using a differential recording mode (Vincent et al. 2016b).

Figure 3. A. Superficial cortical effects of the ES when the space between the two poles of the bipolar electrode is not sufficient $(\sim 0.5 \mathrm{~cm})$ to excite deeper neurons and axons to induce a CACEP. Only local effects can be triggered (DCR) at low frequency. B. When the ES frequency is increased (up to $20 \mathrm{~Hz}$ ) the basal electrophysiological level may be hyperpolarized progressively and the DCR may be attenuated thereby perturbing the functional connectivity of the whole network.

B. Deeper cortical effects of the ES when the space between the two poles is sufficiently large $(\sim 1 \mathrm{~cm})$ and current is increased $(\sim[10 ; 20] \mathrm{mA})$ like when using two electrodes of 
a subdural ECoG grid in epilepsy. The electrical field lines vehicle a sufficient charge in the deeper cortical layer to excite neurons or axons with long range projections to more distant cortical areas, and thereby induce a CACEP. 